\title{
Beyond your very eyes: eye movements are necessary, not sufficient
}

\author{
Ellen M Kok ${ }^{1}$ (D) \& Halszka Jarodzka ${ }^{2,3}$
}

Editor - In a response to our paper 'Before your very eyes, the values and limitations of eye tracking for medical education', ${ }^{1}$ Smith and colleagues discuss two additional critical limitations of eye-tracking methodology ${ }^{2}$ : (i) looking at something does not equal (consciously) processing it, and (ii) changes in processes do not necessary translate into changes in performance. We argue that these are characteristics instead of limitations of eye tracking.

To address the first point, the unique feature of eye tracking is that we can objectively and nonintrusively measure what information entered the human cognitive system. ${ }^{3}$ Eye tracking measures the positioning of light on the fovea, which is the only part of the eye that can process details and colour. Such entrance does not guarantee further cognitive processing, even though that is what happens most of the time. ${ }^{4}$ That is, eye tracking does not directly measure what information is processed, but it

${ }^{1}$ Department of Educational Development and Research, School of Health Professions Education, Maastricht University, Maastricht, The Netherlands

${ }^{2}$ Welten Institute, Research Centre for Learning, Teaching and Technology, Open University of the Netherlands, Heerlen, The Netherlands

${ }^{3}$ Humanities Laboratory, Lund University, Lund, Sweden

Correspondence: Ellen Kok, School of Health Professions Education, Department of Educational Development and Research, Maastricht University, PO Box 616, 6200 MD Maastricht, The Netherlands. Tel: +31 43388576 ;

E-mail: e.kok@maastrichtuniversity.nl indicates what can be processed. Triangulation with other measures, such as verbal protocols, helps to support the interpretation of eye-tracking data. That said, what we can validly determine from eye tracking alone is what was not looked at and, as a result, has hardly a chance of being processed, falling at best onto the periphery of the eye's retina. Spoken in the terminology of logical empiricism, looking at information is a necessary condition for it being processed cognitively; it is, however, not sufficient.

To the second point, we have experienced exactly this issue in our own research: We found that training medical students to systematically inspect radiographs made them 'look at' more of an image (measured via eye tracking) but not 'detect' (i.e. report) more abnormalities. ${ }^{5}$ In this study, eye tracking provided us with invaluable information: that training in a systematic viewing strategy does change viewing behaviour but does NOT change diagnostic performance. The implication is that in this specific case, we should focus our efforts on training students in what abnormalities look like rather than focusing on better viewing strategies. Eye tracking provided unique insight into the root cause of the problem, something that a diagnostic performance test alone could not have.

Like every research technique, eye tracking has its strengths, but also its limitations. Together with verbal data (how?), eye-tracking data (what?) can provide unique insights by helping us detect where exactly the challenges lie within a specific task.

\section{REFERENCES}

1 Kok EM, Jarodzka H. Before your very eyes: the value and limitations of eye tracking in medical education. Med Educ 2017;51 (1):114-22.

2 Smith S, O'Tuathaigh C, Henn P. Behind your very eyes: a response to Kok and Jarodzka. Med Educ 2017; https://doi.org/10.1111/medu. 13341

3 Atkinson RC, Shiffrin RM. Human memory: a proposed system and its control processes. Psychol Learn Motiv 1968;2:89-195.

4 Just MA, Carpenter PA. A theory of reading: from eye fixations to comprehension. Psychol Rev 1980;87:329.

5 Kok EM, Jarodzka H, de Bruin $\mathrm{ABH}$, BinAmir HAN, Robben SGF, van Merriënboer JJG. Systematic viewing in radiology: seeing more, missing less? Adv Health Sci Educ 2016;21:189-205. 